PAPER

\title{
Amyloid mediates the association of apolipoprotein E e4 allele to cognitive function in older people
}

\author{
D A Bennett, J A Schneider, R S Wilson, J L Bienias, E Berry-Kravis, S E Arnold
}

J Neurol Neurosurg Psychiatry 2005;76:1194-1 199. doi: 10.1136/jnnp.2004.054445

See end of article for authors' affiliations

Correspondence to Dr D A Bennett, Rush Alzheimer's Disease Center, Armour Academic Center, 600 South Paulina, Suite 1028; Chicago, IL. 60612; dbennett@rush. edu

Received

19 September 2004

In revised form

18 November 2004

Accepted 4 January 2005
Background: The neurobiological changes underlying the association of the apolipoprotein E (APOE) e4 allele with level of cognition are poorly understood.

Objective: To test the hypothesis that amyloid load can account for (mediate) the association of the APOE e4 allele with level of cognition assessed proximate to death.

Methods: There were 44 subjects with clinically diagnosed Alzheimer's disease and 50 without dementia, who had participated in the Religious Orders Study. They underwent determination of APOE allele status, had comprehensive cognitive testing in the last year of life, and brain autopsy at death. The percentage area of cortex occupied by amyloid beta and the density of tau positive neurofibrillary tangles were quantified from six brain regions and averaged to yield summary measures of amyloid load and neurofibrillary tangles. Multiple regression analyses were used to examine whether amyloid load could account for the effect of allele status on level of cognition, controlling for age, sex, and education.

Results: Possession of at least one APOE e4 allele was associated with lower level of cognitive function proximate to death $(p=0.04)$. The effect of the e4 allele was reduced by nearly $60 \%$ and was no longer significant after controlling for the effect of amyloid load, whereas there was a robust inverse association between amyloid and cognition $(p=0.001)$. Because prior work had suggested that neurofibrillary tangles could account for the association of amyloid on cognition, we next examined whether amyloid could account for the effect of allele status on tangles. In a series of regression analyses, e4 was associated with density of tangles $(p=0.002)$, but the effect of the e 4 allele was reduced by more than $50 \%$ and was no longer significant after controlling for the effect of amyloid load.

Conclusion: These findings are consistent with a sequence of events whereby the e4 allele works through amyloid deposition and subsequent tangle formation to cause cognitive impairment.
A lzheimer's disease $(\mathrm{AD})$ is a complex disease with multiple genetic and environmental risk factors. To cause cognitive impairment, these risk factors must interact with the types, quantities, and distributions of pathological lesions in the brain. For example, it is likely that some risk factors cause cognitive impairment by enhancing amyloid production or fibrillogenesis, decreasing amyloid clearance, or by enhancing tau phosphorylation. As $\mathrm{AD}$ pathology often coexists with other common age related pathology that also contributes to cognitive impairment, such as cerebral infarcts and Lewy bodies, ${ }^{1}$ it is likely that other risk factors cause cognitive impairment by increasing the number or density of these pathological lesions. Finally, it is likely that still other risk factors lead to cognitive impairment by causing neurodegenerative changes independent of the pathological makers of common age related diseases. Understanding the neurobiology that links risk factors to cognitive impairment has important implications for disease treatment and prevention.

The presence of one or more apolipoprotein E (APOE) e4 alleles is a major genetic risk factor for $\mathrm{AD}^{2-4}$ The neurobiological changes in the brain that account for the association of allele status with disease risk remain poorly understood. Some clinicopathological studies report an association between the e4 allele and neuritic plaques and neurofibrillary tangles, ${ }^{5-7}$ although other studies did not find this association. ${ }^{8}{ }^{9}$ Several studies also report a relationship between the e 4 allele and amyloid deposition, ${ }^{10-16}$ raising the possibility that amyloid deposition accounts for the association of allele status with cognitive function.

We used data from the Religious Orders Study, a longitudinal clinicopathological study of aging and $\mathrm{AD}$, to investigate whether amyloid mediates the association of APOE allele status with level of cognition. The term "mediate" is used here in the statistical sense, and refers to accounting for the association between allele status and cognition by controlling for amyloid in a regression model. These types of analyses are increasingly being used in behavioural and epidemiological studies in an effort to go beyond simple associations and investigate potential intermediate steps in a causal chain that link risk factors to adverse clinical outcomes. ${ }^{17}{ }^{18}$ In this case, it addresses the extent to which amyloid deposition represents a key step in the causal chain linking allele status with cognitive impairment. The approach is similar to identifying mediating factors that account for the association between hypertension and ischemic stroke. For example, one mechanism by which hypertension leads to stroke is cerebral atherosclerosis. It would be expected that a change in the relationship between hypertension and stroke would be observed after controlling for carotid atherosclerosis in an analysis, as atherosclerosis is an important link in the causal chain. We have used these types of analyses to investigate how postmortem indices work together ${ }^{1920}$ and with a variety of risk factors ${ }^{21-25}$ to lead to cognitive impairment.

We previously reported that measures of plaques and tangles identified with a silver stain mediated the association of allele status to level of cognition. ${ }^{21}$ However, because silver stains are neither as sensitive as immunohistochemistry nor as protein specific, these measurements are not ideally suited to addressing the extent to which the association of the e4

Abbreviations: $A D$, Alzheimer's disease; $A P O E$, apolipoprotein $E$; DMSO, dimethyl sulphoxide; PHF, paired helical filament 
allele with cognition can be accounted for by amyloid deposition. The analyses in this study followed a similar structure. We first documented the association of allele status to level of cognition proximate to death. We subsequently tested the hypothesis that amyloid mediates the association of allele status with cognition by adding a term for amyloid to the model. If amyloid is an important mediating variable, the association between allele status and level of cognition should be reduced. If allele status was related to cognition through some other mechanism, then adding a term for amyloid to the model would not influence its association with cognition. As we previously provided evidence that tangles mediate the association of amyloid on cognition, ${ }^{19}$ we next conducted a similar set of analyses to determine whether amyloid mediated the effect of allele status on tangles. Together, these analyses should provide evidence in favour of or against a sequence of events whereby e 4 works through amyloid deposition and subsequent tangle formation to cause cognitive impairment, as outlined in fig 1 . We are unaware of any prior study that has examined the joint effect of allele status and amyloid deposition on level of cognition assessed proximate to death.

\section{METHODS \\ Subjects}

Subjects were older Catholic nuns, priests, and monks participating in the Religious Orders Study (see Acknowledgements). Each participant agreed to an annual clinical evaluation and signed an informed consent and an Anatomical Gift Act donating their brain to Rush investigators at the time of death. The study was approved by the Human Investigations Committee of Rush University Medical Center. Since January 1994, more than 1000 people have enrolled in the study and completed the baseline evaluation. Participation in the annual follow up evaluations exceeds $95 \%$ of survivors, and the autopsy rate exceeds $90 \%{ }^{26}{ }^{27}$

\section{Clinical evaluation}

All subjects underwent a uniform structured clinical evaluation that included a medical history, neurological examination, review of a brain scan when available, and 21 cognitive performance tests (see below). Results of the cognitive tests were reviewed by a board certified neuropsychologist, and participants were evaluated in person by a physician with expertise in the evaluation of older people. Based on this evaluation, participants were classified with respect to $\mathrm{AD}$ and other common conditions with the potential to impact cognitive function. Details of the clinical evaluation have been previously described. ${ }^{2026}$ Follow up evaluations, identical in all essential details, were performed annually by examiners blinded to previously collected data. At the time of death, all available clinical data were reviewed and a summary diagnostic opinion was rendered regarding the most likely clinical diagnosis at the time of death.

The 21 cognitive performance tests assessed a broad range of cognitive abilities that are commonly affected by aging and $\mathrm{AD}$. Details of the cognitive tests have been previously reported. ${ }^{26}{ }^{27}$ A global cognitive summary measure was used in analyses to minimise floor and ceiling effects and other

$$
\text { ApoE e4 }- \text { Amyloid }- \text { Tau tangles }-\begin{aligned}
& \text { Cognitive } \\
& \text { function }
\end{aligned}
$$

Figure 1 Hypothesised sequence of events that links APOE allele status to level of cognition proximate to death. If the sequence is correct, then amyloid should mediate that association of allele status on cognition and on tangles, but tangles would not mediate the association of allele status on cognition. We have previously shown that tangles mediate the association of amyloid on cognition. ${ }^{19}$ sources of measurement error. It was constructed by converting the raw scores from 19 individual tests to $\mathrm{Z}$ scores, using the mean and standard deviation from the baseline evaluation of all participants, and averaging the $\mathrm{z}$ scores. To have a valid summary score, at least half of the component scores had to be present. Details of the individual cognitive tests and the derivation of the summary measure has been previously reported..$^{26}$

\section{Apolipoprotein E genotyping}

Blood was collected at each participating Religious Order Study site with acid citrate dextrose anticoagulant and stored at room temperature. Lymphocyte separation was performed within 24 hours of collection. DNA was extracted from approximately 2-3 million cells, and genotyping was performed by an investigator blinded to all clinical and postmortem data as previously described. ${ }^{21}$

\section{Tissue preparation and immunocytochemistry}

Brains of deceased subjects were removed and weighed in a standard fashion as described previously. ${ }^{19-21}$ Each brain was cut coronally into $1 \mathrm{~cm}$ thick coronal slabs, immersion fixed in $4 \%$ paraformaldehyde, and placed in graded glycerol/ dimethylsulphoxide (DMSO) in phosphate buffered saline for storage with final dilutions being $20 \%$ glycerol and $2 \%$ DMSO. Tissue from six regions of interest was dissected into $0.5 \mathrm{~cm}$ thick blocks and embedded in paraffin. For the entorhinal cortex proper and the CAl/subiculum, $20 \mu \mathrm{m}$ sections were cut from consecutive $1 \mathrm{~cm}$ slabs throughout its length (up to six blocks per case). For the four neocortical regions, including the midfrontal, inferior temporal, inferior parietal, and primary visual cortices, two blocks were obtained from adjacent $1 \mathrm{~cm}$ slabs and cut into $20 \mu \mathrm{m}$ sections.

Amyloid- $\beta$ was labelled with MO0872, (1:100; Dako, Carpentaria, CA, USA) using diaminobenzidine as the reporter with $2.5 \%$ nickel sulphate to enhance immunoreaction product contrast as previously reported. ${ }^{19}$ MO0872 identifies both the 1-40 and 1-42 length amyloid fragments. All sections were run using identical incubation times on an automated immunohistochemical stainer (Biogenex, San Ramon, CA, USA). All runs included control sections processed without primary antibodies. A systematic random sampling scheme was used to capture images for quantitative analysis of amyloid deposition as previously described. ${ }^{28}$ Briefly, after camera and illumination calibration, 24 bit colour images obtained at each sampling site were obtained and converted to 8 bit greyscale images. Calculation of the percentage area occupied by amyloid- $\beta$ immunoreactive pixels was performed with Object-Image 1.62pl5 (http:// simon.bio.uva.nl/object-image.html). The analysis algorithm segmented the labelled and background compartments of each image using one of two histogram dependent automatic thresholding procedures, iterative self organising data analysis (ISODATA) and triangulation. ${ }^{29}$ A composite summary measure of the percentage area occupied by amyloid- $\beta$ was made by averaging the values for all regions as previously reported. ${ }^{19}$

Paired helical filament (PHF) tau was labelled with AT8 (1:800 in 4\% horse serum; Innnogenex, San Ramon, CA, USA), an antibody specific for phosphorylated tau as described previously. ${ }^{19} 2830$ Neurofibrillary tangles labelled with AT8 have a characteristic appearance and location in the neuronal cell body or as "ghost tangles". All sections were run using identical incubation times on an automated immunohistochemical stainer (Biogenex, San Ramon, CA, USA) in precisely timed runs. All runs included control sections processed without primary antibody. Quantification of tangle density per $\mathrm{mm}^{2}$ was performed with a stereological 
mapping station, which included a Leica DMRBE microscope and a computer (Millennia Mme, Micron Electronic Inc., USA) equipped with StereoInvestigator software (version 5.00; MicroBrightField Inc., Colchester, VT, USA). Briefly, a grid of pre-determined size was randomly placed over the entire region by the software program. At a total magnification of 400, the program directed the motorised stage on the microscope to stop at each intersection point of the grid for sampling. The operator focused through the section depth as the fields were visualised on the video monitor within the superimposed counting frame. All objects within the $150 \times 150 \mu \mathrm{m}$ counting frame that did not touch the exclusion lines of the box (bottom and left sides) were counted. A composite summary measure of the density of tangles was made by averaging the values for all regions as previously reported. ${ }^{19}$

\section{Statistical analysis}

The goal of the statistical analyses was to determine whether amyloid mediates the effect of APOE allele status on level of cognition proximate to death. Once a relationship between two variables such as allele status (the independent variable) and level of cognition (the dependent variable) is established, a mediation hypothesis reflects a causal hypothesis about how a third variable, in this case amyloid, affects their association. ${ }^{17}{ }^{18}$ In general, the third variable can affect the association in one of three ways. It can moderate the association such that an observed relationship may be different at different levels of a third variable (effect modification or interaction), it can mediate the association such that controlling for the third variable can attenuate the effect of the independent variable, or it can confound the association. Mediation and confounding are identical statistically, but can be distinguished on conceptual grounds. ${ }^{17} 18$ In this case, as the association between allele status and cognition is well established, as is the association of measures of $\mathrm{AD}$ pathology with cognition, and as allele status precedes the accumulation of AD pathology, and as evidence from a variety of sources supports a biologically plausible hypothesis linking allele status to amyloid, the results are unlikely to be due to confounding.

A series of multiple regression analyses was conducted to test the hypothesised sequence presented in fig 1. Participants were divided into two APOE subgroups: those with one or more e4 alleles $(\mathrm{e} 2 / 4, \mathrm{e} 3 / 4, \mathrm{e} 4 / 4)$ and those without any e4 alleles $(\mathrm{e} 2 / 2, \mathrm{e} 2 / 3, \mathrm{e} 3 / 3)$. The first analysis documented the association of allele status with level of cognition. We then conducted a second analysis in which we included a term for amyloid load in the regression model to see if it reduced the affect of allele status on cognition as hypothesised. If our hypothesis is correct, we would expect that the association of the e4 allele with cognition would be reduced after controlling for amyloid. Because we previously provided evidence that tangles mediate the association of amyloid to cognition, ${ }^{19}$ we hypothesised that amyloid would mediate the association of allele status with tangles. Therefore, we next examined the association of allele status with tangles. In a second regression model, we added a term for amyloid to see if it reduced the association of the e 4 allele with tangles. We also conducted a similar set of analyses in which tangles were added to a model examining amyloid as a function of allele status. If our hypothesis is correct, we would expect that the association of the e 4 allele with tangles would be attenuated by inclusion of amyloid in the model. By contrast, the association of the e4 allele with amyloid would remain significant after controlling for tangles. All regression models controlled for the potentially confounding effects or age, sex, and education. We have previously used this analytic approach to examine how pathological variables work in concert $^{19} 20$ and with risk factors ${ }^{21-25}$ to cause cognitive impairment. Models were validated both graphically and analytically, and the assumptions were not violated despite some pathological indices being skewed. All analyses were carried out using SAS/STAT software (version 8; SAS Institute Inc., Cary, NC, USA) on a SunUltraSparc workstation. $^{31}$

\section{RESULTS}

Of the 94 four deceased participants in these analyses (40 men, 54 women), 44 met clinical criteria for probable AD and 50 did not have dementia. Subjects with AD were older, had slightly fewer years of education, and had lower scores on the MMSE $^{32}$ and on the measure of global cognitive function proximate to death (table 1 ). One or more e4 alleles were found in 28 (29.8\%) of the subjects including three with e2/4, 22 with e3/4, and three with e4/4; the remaining $66 \mathrm{did}$ not have a e4 allele (seven with e2/3 and 59 with e3/3). The median interval from the last clinical evaluation to brain autopsy was about 8 months. Subjects with AD had about twice as much amyloid and nearly four times as many tangles as those without $\mathrm{AD}$ (table 1).

\section{Amyloid mediates the association of the e4 allele to level of cognition}

We first documented the relation of the APOE e4 allele to level of global cognition proximate to death. In a linear regression model controlling for age, sex, and education, the presence of one or more e4 alleles was associated with cognitive function score of almost half unit lower $(p=0.04)$ (table 2, model 1). When we repeated the analysis with a term for amyloid load added to the model, the effect of the e4 allele was reduced by nearly $60 \%$ and was no longer significant, whereas amyloid had a robust association with cognitive impairment $(p=0.001)$ (table 2, model 2). These data are consistent with a sequence of events whereby amyloid deposition accounts for the association of the e4 allele with cognitive function.

\section{Amyloid mediates the association of the e4 allele to tangles}

We previously reported that tau positive neurofibrillary tangles mediate the association of amyloid with cognition. ${ }^{19}$ If amyloid mediates the relation of the e4 allele with cognitive function, then we hypothesised that it would also mediate the association of allele status with tangles as suggested by the hypothesised sequence outlined in fig 1 . To

Table 1 Selected characteristics of participants with Alzheimer's disease and without dementia

\begin{tabular}{|c|c|c|c|}
\hline Characteristics & No dementia & Probable AD & Total \\
\hline \multicolumn{4}{|l|}{ Demographic } \\
\hline $\mathrm{n}$ & 50 & 44 & 94 \\
\hline Male (\%) & 56.0 & 38.6 & 42.6 \\
\hline $\begin{array}{l}\text { Mean (SD) age at } \\
\text { death }\end{array}$ & $82.3(6.6)$ & $88.1(5.3)$ & $85.0(6.7)$ \\
\hline $\begin{array}{l}\text { Mean (SD) } \\
\text { education }\end{array}$ & $18.7(3.4)$ & $17.3(3.0)$ & $18.1(3.3)$ \\
\hline APOE e4 allele (\%) & $8(16.0 \%)$ & $20(45.5 \%)$ & $28(29.8 \%)$ \\
\hline \multicolumn{4}{|l|}{ Clinical } \\
\hline $\begin{array}{l}\text { Mean (SD) MMSE } \\
\text { score }\end{array}$ & 27.7 (1.9) & $16.1(7.4)$ & $22.2(7.8)$ \\
\hline Mean (SD) GC score & $-0.06(0.54)$ & $-1.82(0.83)$ & $-0.89(1.12)$ \\
\hline \multicolumn{4}{|l|}{ Pathological } \\
\hline $\begin{array}{l}\text { Mean (SD) amyloid } \\
\text { load, \% }\end{array}$ & $1.88(1.85)$ & $3.81(2.47)$ & $2.79(2.36)$ \\
\hline $\begin{array}{l}\text { Mean (SD) tau } \\
\text { tangles } / \mathrm{mm}^{2}\end{array}$ & $3.62(3.71)$ & $14.22(12.38)$ & $8.58(10.31)$ \\
\hline
\end{tabular}


Table 2 Summary of multiple linear regression analyses examining a global measure of cognitive abilities as a function of the e4 allele (model 1), and both the e4 allele and a measure of amyloid load (model 2). All models controlled for age, sex, and education

\begin{tabular}{|c|c|c|c|c|}
\hline \multirow{2}{*}{$\begin{array}{l}\text { Outcome } \\
\text { measure terms }\end{array}$} & \multicolumn{2}{|l|}{ Model 1} & \multicolumn{2}{|l|}{ Model 2} \\
\hline & Estimate (SE) & $p$ value & Estimate (SE) & $p$ value \\
\hline \multicolumn{5}{|l|}{ GC } \\
\hline e4 allele & $-0.432(0.210)$ & 0.04 & $-0.176(0.214)$ & 0.41 \\
\hline Amyloid load & d - & - & $-0.145(0.044)$ & 0.001 \\
\hline
\end{tabular}

test this hypothesis, we first examined the density of tangles as a function of the e4 allele adjusting for age, sex, and education. In these analyses, the presence of one or more e4 alleles was associated with having approximately seven more tangles per $\mathrm{mm}^{2}(\mathrm{p}=0.002)$ (table 3 , model 1 ). When we repeated the analysis with a term for amyloid load added to the model, the effect of the e 4 allele was reduced by more than $50 \%$ and was no longer significant, whereas amyloid had a robust association with tangles $(p<0.001)$. To ensure that the finding was most consistent with the hypothesised sequence, we next considered the opposite sequence, namely that tau tangles mediated the relation of the e4 allele to amyloid. To examine this possibility, we examined amyloid as a function of allele status, adjusting for age, sex, and education. The presence of one or more e4 alleles was associated with an almost $2 \%$ increase in amyloid load $(\mathrm{p}<0.001)$ (table 3, model 1$)$. When a term for tangles was added to the model, the effect of the e4 allele was attenuated, but remained significant $(\mathrm{p}=0.02)$. These data suggest that tangles cannot fully account for the association of the e4 allele with amyloid.

\section{DISCUSSION}

We quantified extracellular deposits of amyloid- $\beta$ peptide plaque deposits and phosphorylated tau immunoreactive neurofibrillary tangles from multiple brain regions from subjects with and without clinically diagnosed $\mathrm{AD}$, and examined a hypothesised sequence of events linking APOE allele status to level of cognition assessed proximate to death (fig 1). We found that amyloid accounted for the association of the e4 allele to level of cognition. Further, we found that amyloid accounted for the association of allele status to tangles, but tangles did not account for the association of the $\epsilon 4$ allele to amyloid. Together with data from a prior study, ${ }^{19}$ these data suggest that the APOE e4 allele is the first step in a sequence of events that works through amyloid deposition and subsequently tangle formation to cause cognitive impairment, as outlined in fig 1.

Table 3 Summary of multiple linear regression analyses

\begin{tabular}{|c|c|c|c|c|}
\hline \multirow{2}{*}{$\begin{array}{l}\text { Outcome } \\
\text { measure terms }\end{array}$} & \multicolumn{2}{|l|}{ Model 1} & \multicolumn{2}{|l|}{ Model 2} \\
\hline & Estimate (SE) & $p$ value & Estimate (SE) & $p$ value \\
\hline \multicolumn{5}{|l|}{ Tau tangles } \\
\hline e4 allele & $6.98(2.21)$ & 0.002 & $3.39(2.13)$ & 0.12 \\
\hline Amyloid load & - & - & $2.04(0.44)$ & $<0.001$ \\
\hline \multicolumn{5}{|l|}{ Amyloid load } \\
\hline e4 allele & $1.76(0.48)$ & $<0.001$ & $1.08(0.46)$ & 0.02 \\
\hline Tau tangles & - & - & $0.10(0.02)$ & $<0.0001$ \\
\hline
\end{tabular}

The first pair of models examined tau tangles as a function of the e 4 allele (model 1) and both the e4 allele and amyloid (model 2). The second set of models examined amyloid load as a function of the e4 allele (model 1) and both the e4 allele and tau tangles (model 2). All models controlled for age, sex, and education.
Understanding how neuropathological indices account for the association of risk factors to cognitive impairment has important implications regarding strategies to delay disease onset. Over the past several years, possession of one or more APOE e4 alleles has emerged as the most important genetic susceptibility factors for $\mathrm{AD}$ among older people. Despite intensive investigation, the neurobiological changes responsible for the association of allele status with the occurrence of clinical disease remains controversial, with some data suggesting that its effect is mediated by an increase in the rate of accumulation of $\mathrm{AD}$ pathology, ${ }^{5-7}$ whereas other data suggest that it may be related to other less specific mechanisms such as neural repair or survival. ${ }^{8} 9334$

$\mathrm{We}^{21}$ and others ${ }^{5-7}$ have reported that allele status is related to $\mathrm{AD}$ pathology as identified with routine histopathology. Further, several studies have found a relation between the e4 allele and amyloid deposition ${ }^{10-13}$ or both amyloid and tau positive tangles. ${ }^{14}{ }^{15}$ By contrast, some studies have found an association of the e4 allele and amyloid deposition but not tau tangles. ${ }^{16}$ The data in this study extend the prior publications not only by showing an association of allele status with amyloid deposition and tangle formation, but also by providing strong evidence that amyloid deposition mediates the association of the e4 allele with tau tangles and ultimately with level of cognition. The term mediate is used in the statistical sense and refers to the fact that accounting for amyloid in analyses markedly attenuates the association of allele status with cognition. This finding does not preclude the possibility that the e4 allele may work through other mechanisms in addition to amyloid, but it implies that the effect sizes of the other variables would be substantially smaller.

APOE is synthesised primarily by astrocytes and microglia in the brain and its main function is thought to be in the regulation of lipoprotein metabolism as in the periphery. ${ }^{35}$ However, preclinical data from a variety of sources suggest that APOE may also be involved in the deposition and/or clearance of amyloid from the brain. APOE appears to enhance spontaneous fibrillogenesis of amyloid in vitro, ${ }^{36}$ and has been reported to form dimeric complexes with soluble amyloid in vivo. ${ }^{37}$ The role of APOE on the deposition of amyloid has also been studied in transgenic animals. ${ }^{38-40}$ When transgenic mice expressing amyloid precursor protein were bred with apoE knockout mice, there was some deposition of non-fibrillar amyloid. However, neuritic dystrophy was seen in association with the amyloid when transgenics were bred with wild type mice possessing the murine apoE gene. Likewise, nonfibrillar amyloid was seen in transgenics bred onto mice expressing human APOE e2. However, severe neuritic dystrophy was seen in association with the amyloid in transgenic mice bred with mice expressing human APOE e3 or e4, with the latter having a greater effect on amyloid plaque formation.

The study has several strengths. APOE allele status was linked to amyloid and tangles, and to level of global cognitive function assessed proximate to death. All analyses were performed on comparable people from a single cohort who came to autopsy following high rates of follow up participation and brain autopsy. This cohort provides controls for other potentially confounding variables such as occupation and lifestyle, and the sample size was sufficiently large to control for other important and potentially confounding variables such as age, sex, and education. Finally, uniform structured procedures were followed, examiners were blinded to previously collected data, and all personnel collecting postmortem, genetic, and clinical data were blinded to all other data, further reducing the potential for bias.

However, the study also has several limitations. We only quantified the fibrillar form of amyloid- $\beta$ and PHF tau 
positive tangles identified by antibodies to a single epitope Different epitopes of amyloid- $\beta$ or tau, or other pathological indices (such as cerebrovascular disease) may also be related to allele status and clinical disease, suggesting additional studies that incorporate these potential mediators. Further, because we only quantified fibrillar amyloid rather than soluble amyloid, it is not clear whether APOE allele status is associated with increased production or fibrillogenesis of amyloid, or reduced clearance. Finally, participants are not representative of the population of the USA as a whole in terms of education and lifestyle. Therefore, the findings will need to be replicated in similar studies of lay people. Most importantly, clinicopathological analyses are inherently cross sectional, and the sequence under investigation can be inferred but not proven. Thus, although we tested an a priori hypothesis supported by the literature, the data must be interpreted with caution. It may be possible in the future to use the longitudinal sequential clinical observations to model the most likely sequence of underlying pathological events, ${ }^{41}$ Nonetheless, these types of analyses can be a powerful approach to identifying the post-mortem indices that account for the association of genetic and environmental risk factors with $\mathrm{AD}$.

\section{ACKNOWLEDGEMENTS}

We are indebted to the hundreds of nuns, priests, and monks from the following American groups participating in the Religious Orders Study: the Archdiocesan priests of Chicago, Dubuque, and Milwaukee; Benedictine Monks, Lisle, IL and Collegeville, MN; Benedictine Sisters of Erie, Erie, PA; Benedictine Sisters of the Sacred Heart, Lisle, IL; Capuchin monks, Appleton, WI; Christian Brothers, Chicago, IL and Memphis, TN; Diocesan priests, Gary, IN; Dominicans, River Forest, IL; Felician Sisters, Chicago, IL; Franciscan Handmaids of Mary, New York, NY; Franciscan monks, Chicago, IL; Holy Spirit Missionary Sisters, Techny, IL; Maryknolls, Los Altos, CA and Maryknoll, NY; Norbertines, DePere, WI; Oblate Sisters of Providence, Baltimore, MD; Passionist monks, Chicago, IL; Presentation Sisters, Dubuque, BVM, IA; Servites, Chicago, IL; Sinsinawa Dominican Sisters, Chicago, IL and Sinsinawa, WI; Sisters of Charity, BVM, Chicago, IL and Dubuque, IA; Sisters of the Holy Family, New Orleans, LA; Sisters of the Holy Family of Nazareth, Des Plaines, IL; Sisters of Mercy of the Americas, Chicago, IL, Aurora, IL, and Erie PA; Sisters of St. Benedict, St. Cloud, and St. Joseph, MN; Sisters of St. Casimir, Chicago, IL; Sisters of St. Francis of Mary Immaculate, Joliet, IL; Sisters of St. Joseph of LaGrange, LaGrange Park, IL; Society of the Divine Word, Techny, IL; Trappist monks, Gethsemani, KY and Peosta, IA; Wheaton Franciscan Sisters, Wheaton, IL.

We thank J Bach, T Colvin, and G Hoganson for coordinating the Religious Orders Study. We also thank B Howard, Rush Alzheimer's Disease Center Laboratory Coordinator, W Bang for analytic programming, G Dombrowski and G Klein for data management, and the staff of the Rush Alzheimer's Disease Center.

\section{Authors' affiliations \\ D A Bennett, J A Schneider, R S Wilson, Rush Alzheimer's Disease Center, USA}

D A Bennett, J A Schneider, R S Wilson, E Berry-Kravis, Department of Neurological Sciences, Rush University Medical Center, Chicago, IL, USA

J L Bienias, Department of Internal Medicine, Rush Institute for Healthy Aging, USA

S E Arnold, The Center for Neurobiology and Behavior, University of Pennsylvania, Philadelphia, PA, USA

This study was supported by National Institute on Aging grants RO1 AG15819, P30 AG10161.

Competing interests: none

\section{REFERENCES}

1 Bennett DA, Schneider JA, Bienias JL, et al. Mild cognitive impairment is related to Alzheimer disease pathology and cerebral infarctions. Neurology in press.
2 Tang MX, Stern Y, Marder K, et al. The APOE-epsilon4 allele and the risk of Alzheimer disease among African Americans, whites, and Hispanics. JAMA 1998;279:751-5

3 Evans DA, Bennett DA, Wilson RS, et al. Incidence of Alzheimer's disease in a biracial urban community: relation to apolipoprotein E allele status. Arch Neurol 2003;60:185-9.

4 Breitner JC, Jarvik GP, Plassman BL, et al. Risk of Alzheimer disease with the epsilon 4 allele for apolipoprotein $\mathrm{E}$ in a population-based study of men aged 62-73 years. Alzheimer Dis Assoc Disord 1998;12:40-4.

5 Sparks DL, Scheff SW, Liu H, et al. Increased density of senile plaques (SP), but not neurofibrillary tangles (NFT), in non-demented individuals with the apolipoprotein E4 allele: comparison to confirmed Alzheimer's disease patients. J Neurol Sci 1996;138:97-104.

6 Ghebremedhin E, Schultz C, Thal DR, Gender and age modify the association between APOE and AD-related neuropathololgy. Neurol 2001;56:1696-701

7 Tiraboschi P, Hansen LA, Masliah E, et al. Impact of APOE genotype on neuropathologic and neurochemical markers of Alzheimer disease. Neurology 2004:62:1977-83.

8 Landen M, Thorsell A, Wallin A, et al. The apolipoprotein E allele epsilon 4 does not correlate with the number of senile plaques or neurofibrillary tangles in patients with Alzheimer's disease. J Neurol Neurosurg Psychiatry 1996:61:352-6.

9 Berg L, McKeel DW Jr, Miller JP, et al. Clinicopathologic studies in cognitively healthy aging and Alzheimer's disease: relation of histologic markers to dementia severity, age, sex, and apolipoprotein E genotype. Arch Neurol 1998:55:326-35.

10 Polvikoski T, Sulkava R, Haltia M, et al. Apolipoprotein E, dementia, and cortical deposition of beta-amyloid protein. N Engl J Med 1995;333:1242-7.

11 Schmechel DE, Saunders AM, Strittmatter WJ, et al. Increased amyloid betapeptide deposition in cerebral cortex as a consequence of apolipoprotein $\mathrm{E}$ genotype in late-onset Alzheimer disease. Proc Natl Acad Sci USA 1993;90:9649-53.

12 McNamara MJ, Gomez-Isla T, Hyman BT. Apolipoprotein E genotype and deposits of Abeta40 and Abeta42 in Alzheimer disease. Arch Neurol 1998;55:1001-4.

13 Gearing M, Mori H, Mirra SS. Abeta-peptide length and apolipoprotein E genotype in Alzheimer's disease. Ann Neurol 1996;39:395-9.

14 Thaker U, McDonagh AM, Iwatsubo T, et al. Tau load is associated with apolipoprotein $\mathrm{E}$ genotype and the amount of amyloid beta protein, Abeta40, in sporadic and familial Alzheimer's disease. Neuropathol Appl Neurobiol 2003;29:35-44.

15 Ohm TG, Scharnagl H, Marz W, et al. Apolipoprotein E isoforms and the development of low and high Braak stages of Alzheimer's disease-related lesions. Acta Neuropathol (Berl) 1999;98:273-80.

16 Mukaetova-Ladinska EB, Harrington CR, Roth $M$, et al. Presence of the apolipoprotein E type epsilon 4 allele is not associated with neurofibrillary pathology or biochemical changes to tau protein. Dement Geriatr Cogn Disord 1997;8:288-95.

17 MacKinnon DP, Krull JL, Lockwood CM. Equivalence of the mediation, confounding and suppression effect. Prev Sci 2000;1:173-81.

18 Victora CG, Huttly SR, Fuchs SC, et al. The role of conceptual frameworks in epidemiological analysis: a hierarchical approach. Int J Epidemiol 1997;26:224-7.

19 Bennett DA, Schneider JA, Wilson RS, et al. Neurofibrillary tangles mediate the association of amyloid load and with clinical Alzheimer's disease and level of cognitive function. Arch Neurol 2004;61:378-84.

20 Schneider JA, Wilson RS, Bienias JL, et al. Cerebral infarctions and the likelihood of dementia from Alzheimer's disease pathology. Neurology 2004:62:1148-55.

21 Bennett DA, Wilson RS, Schneider JA, et al. Apolipoprotein E $\epsilon 4$ allele, Alzheimer's disease pathology, and the clinical expression of Alzheimer's disease. Neurology 2003;60:246-52.

22 Bennett DA, Wilson RS, Schneider JA, et al. Education modifies the relation of $A D$ pathology to cognitive function in older persons. Neurology 2003;60:1909-15

23 Wilson RS, Evans DA, Bienias JL, et al. Proneness to psychological distress and risk of Alzheimer's disease. Neurology 2003;61:1579-85.

24 Bennett DA, Wilson RS, Schneider JA, et al. Cerebral infarctions and the relation of depressive symptoms to level of cognitive function in older persons. Am J Geriatric Psychiatry 2004;12:21 1-19.

25 Wilson RS, Schneider JA, Bienias JL, et al. Depressive symptoms, clinical AD, and cortical plaques and tangles in older persons. Neurology 2003:61:1102-1108.

26 Wilson RS, Beckett LA, Barnes LL, et al. Individual differences in rates of change in cognitive abilities of older persons. Psych Aging 2002;17:179-93.

27 Bennett DA, Wilson RS, Schneider JA, et al. Natural history of mild cognitive impairment in older persons. Neurology 2002;59:198-205.

28 Mitchell TW, Nissanov J, Han LY, et al. Novel method to quantify neuropil threads in brains from elders with or without cognitive impairment. $J$ Histochem Cytochem 2000;48:1627-38.

29 Bezdek JC. A convergence theorem for the fuzzy ISODATA clustering algorithm. IEEE Trans Pattern Anal Mach Intell PAMI 1980;2:1-8.

30 Mitchell TW, Mufson EJ, Schneider JA, et al. Parahippocampal tau pathology in healthy aging, mild cognitive impairment and early Alzheimer's disease. Ann Neurol 2002;51:182-9.

31 SAS Institute. SAS/STAT user's guide, version 8. Cary, NC: SAS Institute, 2000.

32 Folstein MF, Folstein SE, McHugh PR. "Mini-Mental State": A practical method for grading the mental state of patients for the clinician. J Psychiatric Research 1975, 12:189-98 
33 Poirier J. Apolipoprotein E in animal models of CNS injury and in Alzheimer's disease. TINS 1994; 17:525-30.

34 Pitas RE, Ji ZS, Weisgraber KH, et al. Role of apolipoprotein E in modulating neurite outgrowth: potential effect of intracellular apolipoprotein E. Biochem Soc Trans 1998:26:257-62.

35 Mahley RW, Rall SC Jr. Apolipoprotein E: far more than a lipid transport protein. Annu Rev Genomics Hum Genet 2000;1:507-37.

36 Wisniewski T, Castano EM, Golabek A, et al. Acceleration of Alzheimer's fibril formation by apolipoprotein E in vitro. Am J Pathol 1994;145:1030-5.

37 Permanne B, Perez C, Soto C, et al. Detection of apolipoprotein E/dimeric soluble amyloid beta complexes in Alzheimer's disease brain supernatants. Biochem Biophys Res Commun 1997;240:715-20.
38 Bales KR, Verina T, Cummins DJ, et al. Apolipoprotein E is essential for amyloid deposition in the APP(V717F) transgenic mouse model of Alzheimer's disease. Proc Natl Acad Sci USA 1999;96:15233-8.

39 Holtzman DM, Bales KR, Tenkova T, et al. Apolipoprotein E isoformdependent amyloid deposition and neuritic degeneration in a mouse model of Alzheimer's disease. Proc Natl Acad Sci SA 2000;97:2892-7.

40 Holtzman DM, Fagan AM, Mackey B, et al. Apolipoprotein E facilitates neuritic and cerebrovascular plaque formation in an Alzheimer's disease model. Ann Neurol 2000:47:739-747.

41 Beckett LA, Bennett DA, Cochran EJ. Modeling sequential clinical observations and post-mortem data. Cleveland, OH: Biometrika Society, 1994.

\section{NEUROLOGICAL PICTURE}

\section{Thrombus in transit through a patent foramen ovale: paradoxical embolism}

A 54 year old man presented with right sided arm and leg weakness of sudden onset accompanied by progressive shortness of breath, 3 days after discharge following admission to the intensive care unit (ICU) for treatment of his pneumonia. He complained of a painful right calf. On neurological examination he had a right sided hemiparesis and extensor plantar response. Brain computed tomography showed recent left frontal infarction. A ventilation perfusion lung scan was consistent with multiple pulmonary emboli. Subsequently, ultrasound showed right popliteal vein thrombosis. Two dimensional transoesophageal echocardiography revealed a large thrombus, floating in the right atrium, traversing a patent foramen ovale (PFO) into the left atrium (fig 1). Following thoracotomy, the thrombus was extracted and the patent foramen ovale was closed. The patient recovered with no further symptoms.

Our patient probably developed deep venous thrombosis (DVT) during his stay in the ICU. Thereafter pulmonary emboli may have caused pulmonary hypertension, thus producing right-left interatrial shunting followed by lodging of a thrombus in the patent foramen ovale. Embolism to the brain occurred either from the venous source through the foramen or from the trapped thrombus in the foramen.

Paradoxical embolism has been postulated as a potential mechanism for stroke in patients with PFO, but documented cases including a "trapped" thrombus and DVT are rare. In stroke patients with PFO, no difference in primary event rates after treatment with either anticoagulants or aspirin, and relatively low detection rates for DVT were found. ${ }^{12}$ Furthermore, an isolated PFO-that is, not in combination with an atrial septal aneurysm, seems not to herald a substantial increased risk of subsequent stroke or death in stroke patients. ${ }^{34}$ Paradoxical embolism thus, only in part explains ischaemic stroke in PFO patients.

J Raaphorst, E J Wouda Department of Neurology, Sint Lucas Andreas Hospital, Jan Tooropstraat 164, 1061 AE Amsterdam, The Netherlands

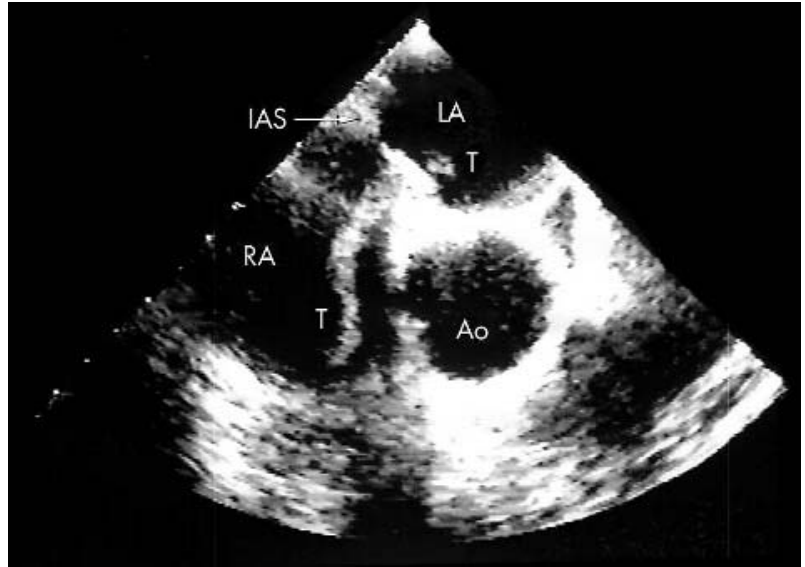

Figure 1 Image of the heart in the oblique plane (50 degrees), at the level of the aortic root. A large thrombus is trapped in a patent foramen ovale and extends into the right and left atrium. T, thrombus; RA, right atrium; LA, left atrium; IAS, interatrial septum; Ao, aorta.

Correspondence to: Dr J Raaphorst, Department of Neurology, Sint Lucas Andreas Hospital, Jan Tooropstraat 164, 1061 AE Amsterdam, The Netherlands; j.raaphorst@slaz.nl

Competing interests: none

\section{References}

1 Homma S, Sacco RL, Di Tullio MR, et al. Effect of medical treatment in stroke patients with patent foramen ovale: Patent Foramen Ovale in Cryptogenic Stroke Study. Circulation 2002;105:2625-31.

2 Lethen H, Flachskampf FA, Schneider R, et al. Frequency of deep vein thrombosis in patients with patent foramen ovale and ischemic stroke or transient ischemic attack. Am J Cardiol 1997;80:1066-9.

3 Mas JL, Arquizan C, Lamy C, et al. Recurrent cerebrovascular events associated with patent foramen ovale, atrial septum aneurysm, or both. N Engl J Med 2001;345:1740-6.

4 Messe SR, Silverman IE, Kizer JR. Practice parameter: recurrent stroke with patent foramen ovale and atrial septal aneurysm: report of the Quality Standards Subcommittee of the American Academy of Neurology. Neurology 2004;62:1042-50. 